In 1929 my attention was drawn to the fact that in the fundus of a young lady who had thrombosis with many haemorrhages, though one could see clearly with the ordinary electric light, the picture by red-free light was very dim. The vitreous, though clear, would not transmit the blue end of the spectrum.

Since then I have seen eight cases, and wherever the haemorrhages were extensive, the vitreous was found to be more or less opaque to red-free light.

Four patients were under 40 years of age. In two there were many haemorrhages, but glaucoma did not supervene, presumably because of youth. In two there were few haemorrhages, and neither was the vitreous stained, nor did glaucoma occur. Five cases were seen in older people. In two the haemorrhages were few, and the vitreous appeared similar to that in the other eye. Glaucoma did not occur.

In three there were many haemorrhages, with definite dimness of the fundus in red-free light. Two developed glaucoma, but died before pain demanded excision. In the third case I had to excise the eye. I was more interested in his retina than in his vitreous, and it did not occur to me to ask a bio-chemist to test the latter for haemoglobin derivatives, as might have been possible.

By red-free light one can see in some cases that not only are there haemorrhages in the retina, there are also haemorrhages on the retina which show up as dark glistening spheres.

The suggestion derived from these few cases is that some substance formed from haemoglobin is absorbed into the vitreous, and if in sufficient quantity alters its light transmitting properties, and may cause a turgescence of the vitreous gel.

Where there are a larger number of cases available for examination one hopes this suggestion may lead to further investigation.

\title{
KANDA'S CURVED SCREW ROLLER FORCEPS (A NEW MODEL OF MODIFICATION FROM KNAPP'S ROLLER FORCEPS)
}

BY

\author{
Dr. KanjI Kanda \\ TAKAO, FORMOSA, JAPAN
}

"Expression of the granules with Knapp's Roller Forceps is" says Professor Komoto, "an international method of treatment of Trachoma." Indeed, Knapp's roller forceps, with its long history of existence since first devised by Dr. Knapp, is the most excellent instrument among those of the same design, so that it 
is still used widely everywhere nearly all the world over with the highest admiration. But as for the mode of application, there may be a few different ways of handling the instrument among different ophthalmic practitioners, mostly according to the positions of the patients under operation.

In my country, also including Formosa, it is usual for the patient to lie down upon the bed for the operation with his face looking upward, and the operator stands behind him, looking over the head of the patient down at his face. This is the most common position of operator and the patient who is undergoing the operation for trachoma with Knapp's roller forceps. In this position, therefore, Knapp's roller forceps is deeply inserted into the everted conjunctival fornix (of upper lid) and pulled out not downward as it is usually shown in the text-books, but upward and forward because of the free margin of the everted conjunctiva being directed toward the ceiling of the operation room; so that the handling motion of the forceps must be done upward and downward in direction, with the straight legs of Knapp's forceps being grasped by the hand of operator quite vertically to the frontal plane of the face of the patient. This makes an inevitable obstacle to the sight of operator, because the straight legs or handles of Knapp's roller forceps, together with the grasping hand of operator stand at the line of sight of the latter and thus narrow the field of vision under operation. And this obstacle is quite common to every practitioner so far as he takes such a position as above mentioned.

The author, therefore, has devised, a new model of reasonable modification from Knapp's roller forceps, in which the legs or handles of the forceps are bent in curves at right angles at about $1.5 \mathrm{~cm}$. above the rollers. This new type of forceps is very convenient for use, especially when the operator stands behind the patient during operation. When this forceps is in use, the curved handles, being grasped horizontally in the manner of fiddlestick grasping of surgical knives, are parallel to the frontal plane of the face of the patient. And so the grasping hand of the surgeon is quite aside the line of sight of operator, so that it does not obstruct the sight of the latter, but leaves an ample space of sight for the operation.

On the other hand, the author has made screw rollers and attached them to the curved handles of his design, in which the winding of the screws is in opposite direction in each roller. Therefore, the rolling of these screw rollers is much easier than in those of Knapp's roller forceps. As, in Knapp's roller forceps, the rollers are, as everyone knows, grooved horizontally along the long axis of them and perpendicular to the direction of pulling the handles, so that they are not uncommon to be pulled out 
without rolling, owing to the large mass of granules or of the hypertrophied conjunctiva that is squeezed between them, which damages the conjunctiva unnecessarily. While in the author's forceps, because of the lightness of the rolling motion of the screw rollers with simultaneous completeness of expression of the

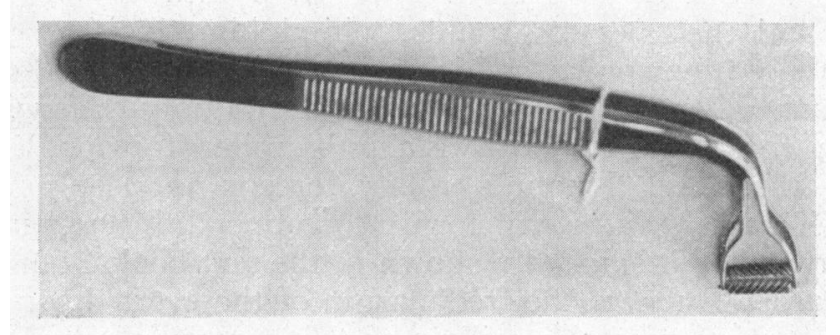

FIG. 1.

Kanda's Curved Screw Roller Forceps.

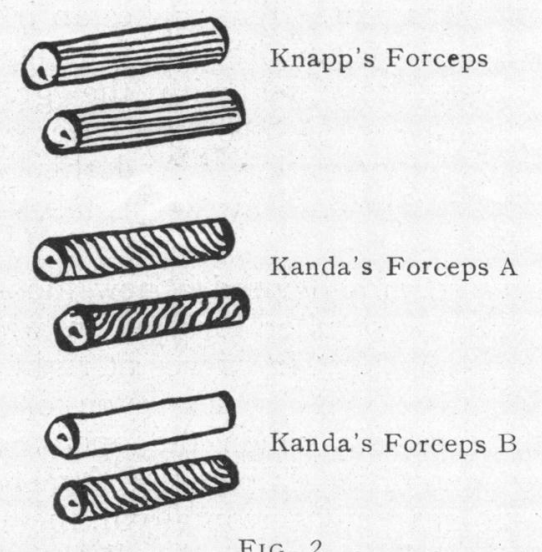

FIG. 2.

The Rollers of Knapp's and Kanda's Forceps.

granules, the proper ability of roller forceps is fully utilized. The author used himself his newly devised forceps with highly successful results. And he calls them "Kanda's Curved Screw Roller Forceps," type A. For he has also devised type B. But in this form only one of the two rollers has screw grooves, and the other one has no grooves, but is only a smooth cylindrical roller of the same size. This latter type of forceps is very useful for the same purpose as the former one, especially for those who have many granules in the conjunctival fornix, and relatively little or no granules in the palpebral conjunctiva. In these cases, 
the B type of forceps, with its smooth roller applied to the intact side of the conjunctiva, is used with much success in expressing the granules of the fornix without harming the palpebral conjunctiva. The author, therefore, wishes every practitioner may have these two of the forceps and take any one of them for the use of operation according to the condition of granules.

As for the author, his own way of expression of trachoma with his newly devised curved screw roller forceps is always accompanied by Kaining's massage and polishing of the conjunctiva with the glass rod. This method brings about many successful results. The curved screw roller forceps of Kanda is manufactured as well as sold by Handaya Medical Instruments Manufacturing Co., Tokio. It has been very successful in his hands and so the author believes no one can be disappointed by the use of this new type of curved screw roller forceps. (October 9, 1931.)

\title{
A SEVENTEENTH CENTURY LETTER DEALING WITH OPHTHALMIC MATTERS, ETC.
}

$$
\text { BY }
$$

\author{
R. R. JAMES \\ LONDON
}

A TRANSCRIPT of the letter here published was sent to me recently. It seems of sufficient interest to be printed in this journal.

Add. MS. 29244, fo. 10.

"Good Mr. Powers,

Because this affect proceedes from accidentall distemper rather than any naturall affluxion of humour, it had bene good to have opened a veyne, but nether $\mathrm{y}^{\mathrm{r}}$ yeares nor $\mathrm{y}^{\mathrm{s}}$ time of $\mathrm{y}^{\mathrm{e}}$ yeare are propitious, therefore if you find any disordered heates, I advise you to $y^{e}$ use of barly water made with liquerice anise seedes etc. I have directed you four thinges none not physicall as $\left(\mathrm{y}^{\mathrm{t}}\right.$ word is comonly taken) but only to rectify $\mathrm{y}^{\mathrm{r}}$ serous humour $\&$ voyd it by urine $w^{c h}$ is $y^{e}$ intention of $y^{e}$ pills to be taken at nights. I love no much tampering with thinges blowne into ye eye but now white sugar candy used as you mention can do no hurt. Therefore you may be bold wth it. I heare yor Dr there is much offended $w^{t}$ me for ... ying agt him. I pray stop ye rumor I assure you I have bene ever silent in ye censure of wandering practitionours \& so of him, but for a few words I inserted into a 